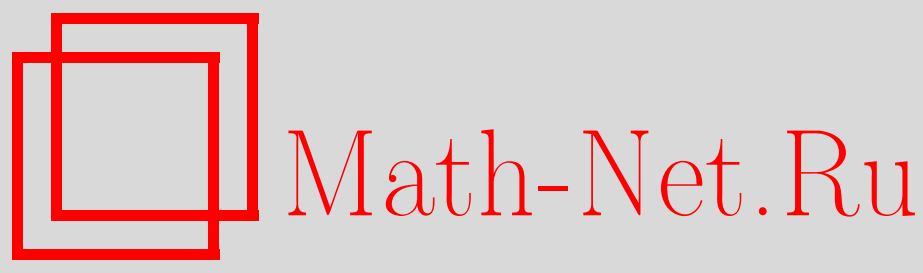

В. Л. Верещагин, Явные решения интегрируемой граничной задачи для двумерной цепочки Тоды, ТMФ, 2010, том 165, номер 1, 25-31

DOI: https://doi.org/10.4213/tmf6561

Использование Общероссийского математического портала Math-Net.Ru подразумевает, что вы прочитали и согласны с пользовательским соглашением http://www . mathnet.ru/rus/agreement

Параметры загрузки:

IP : 54.209 .52 .79

26 апреля 2023 г., $17: 57: 41$

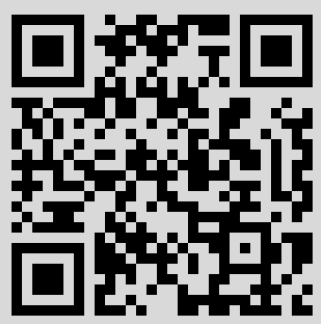




\title{
ЯВНЫЕ РЕШЕНИЯ ИНТЕГРИРУЕМОЙ ГРАНИЧНОЙ ЗАДАЧИ ДЛЯ ДВУМЕРНОЙ ЦЕПОЧКИ ТОДЫ
}

\begin{abstract}
Для получения явного решения интегрируемой граничной задачи для двумерной цепочки Тоды используется метод обратной задачи рассеяния. Свойство интегрируемости интерпретируется в терминах соответствующей линейной задачи, уравнения Гельфанда-Левитана-Марченко и процедуры одевания. Простейшие начальные решения граничной задачи путем одевания переходят в новые нетривиальные решения.
\end{abstract}

Ключевые слова: цепочка Тоды, интегрируемая граничная задача, процедура одевания.

\section{1. ВВЕДЕНИЕ}

Двумерное обобщение цепочки Тоды

$$
u_{x y}(n)=w(n-1)-w(n)
$$

где $w(n)=e^{u(n)-u(n+1)}, u(n)=u(n, x, y), n \in \mathbb{Z}$, было предложено в работе [1] вместе с соответствующими представлением Лакса и набором интегралов движения. В работе [2] содержится описание применения метода обратной задачи рассеяния (МОЗР) к цепочке (1.1): исследована спектральная задача, решена задача восстановления потенциала, а также описаны многосолитонные решения. Однако применение МОЗР оказалось в большой степени затруднено тем фактом, что цепочка (1.1) является объектом размерности $2+1$ : есть зависимость от времени и двух пространственных переменных, одна из которых играет роль целочисленного номера узла решетки. Тем не менее в работах [3], [4] был предложен достаточно богатый набор решений для цепочки (1.1). Далее, с развитием теории интегрируемых граничных задач и ее распространением на многомерные модели [5] стало актуальным применение этого метода к двумерной цепочке Тоды.

Обычно приложения имеют дело с математическими моделями, ассоциированными с граничными задачами в большей степени, нежели с задачами Коши, поэтому вопрос о явном решении таких граничных задач представляется актуальным. Однако далеко не каждая граничная задача совместима со схемой МОЗР в смысле

${ }^{*}$ Институт математики с ВЦ УНЦ РАН, Уфа, Россия. E-mail: v_vereschagin@mail.ru 
интегрируемости. Работа [6], в частности, посвящена ответу на вопрос о корректной формулировке понятия интегрируемости граничной задачи для цепочки (1.1) на диске.

Целью настоящей работы является решение интегрируемой граничной задачи для цепочки Тоды на диске и получение явных формул для соответствующих решений. В разделе 2 описываются основные атрибуты интегрируемости для цепочки (1.1): операторное представление Лакса, метод одевания, определение интегрируемости граничного условия. В разделе 3 упомянутые инструменты применяются к цепочке (1.1): описано уравнение Гельфанда-Левитана-Марченко (ГЛМ), найдено его ядро и определены условия, налагаемые на это ядро фактом интегрируемости граничной задачи. Раздел 4 содержит явные формулы для решений системы ГЛМ, отвечающих интегрируемому граничному условию и простейшему начальному потенциалу. Описана процедура построения сложных формул для решений, которые условно назовем $N$-солитонными, и приведена численная картина для случая $N=1$.

\section{2. ПРИМЕНЕНИЕ МОЗР}

Использование МОЗР в отношении двумерной цепочки Тоды в основном базируется на следующем простом факте: нелинейное уравнение (1.1) является условием совместности системы двух линейных уравнений

$$
\begin{aligned}
& \Psi_{x}(n)=u_{x}(n) \Psi(n)+\Psi(n-1)=L \Psi(n), \\
& \Psi_{y}(n)=w(n) \Psi(n+1)=A \Psi(n),
\end{aligned}
$$

так что нелинейная цепочка (1.1) может быть записана в форме коммутаторного представления

$$
\left[\partial_{x}-A, \partial_{y}-L\right]=0,
$$

где $\partial_{x}=\partial / \partial x,[$,$] - коммутатор. Напомним, следуя работе [3], что одним из спосо-$ бов получения решений уравнения (1.1) является метод одевания Захарова-Шабата. Для этого введем оператор $\widehat{F}$ такой, что

$$
\begin{gathered}
(\widehat{F} \Psi)(n)=\sum_{k=-\infty}^{\infty} F(n, k) \Psi(k), \\
{\left[\partial_{x}-A, F\right]=0, \quad\left[\partial_{y}-L, F\right]=0 .}
\end{gathered}
$$

Предположим, что $\widehat{F}$ допускает треугольную факторизацию:

$$
1+\widehat{F}=(1+\widehat{K})^{-1}\left(1+\widehat{K}^{-}\right),
$$

где

$$
(\widehat{K} \Psi)(n)=\sum_{j=n}^{\infty} K(n, j) \Psi(j), \quad\left(\widehat{K}^{-} \Psi\right)(n)=\sum_{j=-\infty}^{n-1} K^{-}(n, j) \Psi(j) .
$$

Теперь рассмотрим операторы $L^{1}$ и $A^{1}$, определенные следующими условиями:

$$
\begin{aligned}
& \left(\partial_{x}-A^{1}\right)(1+K)=(1+K)\left(\partial_{x}-A^{0}\right), \\
& \left(\partial_{y}-L^{1}\right)(1+K)=(1+K)\left(\partial_{y}-L^{0}\right) .
\end{aligned}
$$


Они удовлетворяют коммутационному соотношению (2.2) и обладают разностной структурой, аналогичной структуре операторов $L^{0}$ и $A^{0}$. Коэффициенты операторов $L^{1}$ и $A^{1}$ могут быть найдены из условий (2.6), таким образом, функции $u^{1}(n)$ являются новыми решениями цепочки Тоды.

Для отыскания матрицы $K$ необходимо решить аналог уравнения ГЛМ, возникающий из соотношений $(2.4),(2.5)$ :

$$
K(n, m)+F(n, m)+\sum_{k=n}^{\infty} K(n, k) F(k, m)=0, \quad m \geqslant n .
$$

Итак, процедура одевания выглядит следующим образом: стартовав с простого потенциала $u^{0}(n)$, решаем линейные уравнения (2.3) для ядра $F(n, m)$. Затем подставляем $F(n, m)$ в уравнение ГЛМ $(2.7)$ и находим матрицу $K(n, m)$, которая с помощью формулы (2.6) позволяет получить новые решения нелинейной цепочки Тоды (1.1).

Нашей задачей является выбор среди "одетых" решений тех, которые удовлетворяют интегрируемому граничному условию [6], [7]. Поэтому потребуется следующее определение.

ОПРЕДЕЛЕНИЕ. Пусть нелинейное уравнение допускает два разных представления Лакса, так что

$$
\left\{Y_{x}=L Y, Y_{y}=A Y\right\}, \quad\left\{\widehat{Y}_{x}=\widehat{L} \widehat{Y}, \widehat{Y}_{y}=\widehat{A} \widehat{Y}\right\} .
$$

Тогда граничное условие $\Omega(u)=0$ на границе $x=0$ называется интегрируемым, если существует линейный дифференциальный (разностный) оператор $B$ такой, что на $x=0$ функция $\widehat{Y}=B Y$ решает уравнение $\widehat{Y}_{y}=\widehat{A} \widehat{Y}$ для любого решения $Y$ уравнения $Y_{y}=A Y$ при условии выполнения граничного условия $\Omega(u)=0$.

\section{3. ИНТЕГРИРУЕМАЯ ГРАНИЧНАЯ ЗАДАЧА И МОЗР}

Выберем окружность фиксированного радиуса $a$ на плоскости $(x, y)$ в качестве границы для нашей задачи. Перепишем цепочку Тоды в полярных координатах $(r, \theta)$ :

$$
u_{r r}(n)+\frac{1}{r} u_{r}(n)+\frac{1}{r^{2}} u_{\theta \theta}(n)=w(n-1)-w(n),
$$

$w(n)=e^{u(n)-u(n+1)}$, а также соответствующую линейную задачу:

$$
\begin{aligned}
& \Psi_{r}(n)=A \Psi(n), \\
& \Psi_{\theta}(n)=L \Psi(n),
\end{aligned}
$$

где

$$
\begin{aligned}
& A_{n m}=\frac{e^{i \theta}}{2} \delta_{n, m-1}-\frac{1}{2}\left(u_{r}(n)-\frac{i}{r} u_{\theta}(n)\right) \delta_{n, m}-\frac{e^{-i \theta}}{2} w(n-1) \delta_{n-1, m}, \\
& L_{n m}=\frac{i r e^{i \theta}}{2} \delta_{n, m-1}-\frac{i r}{2}\left(u_{r}(n)-\frac{i}{r} u_{\theta}(n)\right) \delta_{n, m}+\frac{i r e^{-i \theta}}{2} w(n-1) \delta_{n-1, m},
\end{aligned}
$$


и определим процедуру одевания в полярных координатах. Линейные уравнения (2.3) для ядра $F(n, m)$ системы ГЛМ выглядят следующим образом:

$$
\begin{aligned}
\frac{\partial}{\partial r} F_{n m}= & -\frac{e^{-i \theta}}{2} w(n-1) F_{n-1, m}-\frac{1}{2}\left(u_{r}(n)-\frac{i}{r} u_{\theta}(n)\right) F_{n m}+ \\
& +\frac{e^{i \theta}}{2}\left(F_{n+1, m}-F_{n, m-1}\right)+\frac{1}{2}\left(u_{r}(m)-\frac{i}{r} u_{\theta}(m)\right) F_{n m}+ \\
& +\frac{e^{-i \theta}}{2} w(m) F_{n, m+1}, \\
\frac{\partial}{\partial \theta} F_{n m}= & -\frac{i r e^{-i \theta}}{2} w(n-1) F_{n-1, m}-\frac{i r}{2}\left(u_{r}(n)-\frac{i}{r} u_{\theta}(n)\right) F_{n m}+ \\
& +\frac{i r e^{i \theta}}{2}\left(F_{n+1, m}-F_{n, m-1}\right)+\frac{i r}{2}\left(u_{r}(m)-\frac{i}{r} u_{\theta}(m)\right) F_{n m}- \\
& -\frac{i r e^{-i \theta}}{2} w(m) F_{n, m+1} .
\end{aligned}
$$

Интересующие нас решения отвечают ядру $F_{n m}=\Phi(n) \widehat{\Phi}(m)$. Подставим его в систему (3.6) и убедимся, что функция $\Phi$ является решением линейной системы (3.3), т.е. $\Phi(n)=\Psi(n)$, и функция $\widehat{\Phi}(n)=\widehat{\Psi}(n)$ есть решение следующей системы, в некотором смысле сопряженной к $(3.3)$ :

$$
\begin{aligned}
& \widehat{\Psi}_{r}(n)=-\frac{e^{i \theta}}{2} \widehat{\Psi}(n-1)+\frac{1}{2}\left(u_{r}(n)-\frac{i}{r} u_{\theta}(n)\right) \widehat{\Psi}(n)+\frac{e^{-i \theta}}{2} w(n) \widehat{\Psi}(n+1), \\
& \widehat{\Psi}_{\theta}(n)=-\frac{i r e^{i \theta}}{2} \widehat{\Psi}(n-1)+\frac{i r}{2}\left(u_{r}(n)-\frac{i}{r} u_{\theta}(n)\right) \widehat{\Psi}(n)-\frac{i r e^{-i \theta}}{2} w(n) \widehat{\Psi}(n+1) .
\end{aligned}
$$

В этом случае нужное решение уравнения ГЛМ (2.7) имеет следующий вид:

$$
K(n, m)=-\frac{\Psi(n) \widehat{\Psi}(m)}{1+\sum_{j=n}^{\infty} \Psi(j) \widehat{\Psi}(j)} .
$$

Процедура одевания исходного решения $u^{0}(n)$ использует формулу (3.9) и линейное уравнение (2.6), из которого следует уравнение для коэффициентов операторов $A^{0}, A^{1}$ :

$$
\begin{aligned}
K_{r}(n, m)+\frac{e^{-i \theta}}{2} w^{1}(n-1) K(n-1, m)+\frac{1}{2}\left(u_{r}^{1}(n)-\frac{i}{r} u_{\theta}^{1}(n)\right) K(n, m)- \\
\quad-\frac{e^{i \theta}}{2} K(n+1, m)+\frac{e^{i \theta}}{2} K(n, m-1)-\frac{1}{2}\left(u_{r}^{0}(m)-\frac{i}{r} u_{\theta}^{0}(m)\right) K(n, m)- \\
\quad-\frac{e^{-i \theta}}{2} w^{0}(m) K(n, m+1)-\frac{1}{2}\left(u_{r}^{0}(n)-\frac{i}{r} u_{\theta}^{0}(n)\right) \delta_{n m}-\frac{e^{-i \theta}}{2} w^{0}(n-1) \delta_{n-1, m}+ \\
\quad+\frac{1}{2}\left(u_{r}^{1}(n)-\frac{i}{r} u_{\theta}^{1}(n)\right) \delta_{n m}+\frac{e^{-i \theta}}{2} w^{1}(n-1) \delta_{n-1, m}=0,
\end{aligned}
$$

где $w^{j}(n)=e^{u^{j}(n)-u^{j}(n+1)}, j=0,1$. При $m=n-1$ это дает уравнение

$$
w^{1}(n-1) K(n-1, n-1)-w^{0}(n-1) K(n, n)-w^{0}(n-1)+w^{1}(n-1)=0
$$


и формулу для “одетого” решения:

$$
u^{1}(n)=u^{0}(n)-\ln (1+K(n, n)) .
$$

Нетрудно убедиться, что система (3.7), (3.8) совместна при условии (3.1), поэтому она задает дополнительное коммутативное представление для двумерной цепочки Тоды. Таким образом, наличие двух $L, A$-пар для цепочки (3.1) позволяет использовать приведенное выше определение для формулировки условия интегрируемости граничной задачи в виде существования линейного соотношения между решениями уравнений (3.3) и (3.8) на границе:

$$
\widehat{\Psi}(n)=B(n) \Psi(n),
$$

где $B(n)$ - некоторый разностный оператор. Конкретный вид оператора $B(n)$ и граничного условия может быть получен путем подстановки соотношения (3.11) в уравнение (3.8) при выполнении уравнения (3.3). Собираем коэффициенты при функциях $\Psi(n-1), \Psi(n), \Psi(n+1)$ и приходим к следующему: уравнение

$$
\left.u_{r}(n)\right|_{r=a}=\frac{2 n}{a}+g(\theta)
$$

определяет интегрируемое граничное условие (см. работу [6]), $g(\theta)$ - произвольная функция,

$$
B(n)=(-1)^{n} e^{2 i n \theta+u^{0}(n)+i a \int g(\theta) d \theta} .
$$

Теперь опишем процедуру отыскания явных решений интегрируемой граничной задачи (1.1), (3.12).

1. Для простого исходного потенциала $u^{0}(n)$ решим линейные уравнения (3.3), (3.8).

2. Наложим граничные условия $(3.11),(3.13)$ на полученные функции $\Psi(n), \widehat{\Psi}(n)$ и с помощью формулы $F(n, m)=\Psi(n) \widehat{\Psi}(m)$ получим ядро уравнения ГЛМ, отвечающее нашей граничной задаче.

3. Формулы (3.9) и (3.10) позволяют получить явный вид новых "одетых" решений.

\section{4. ЯВНЫЕ ФОРМУЛЫ ДЛЯ РЕШЕНИЙ}

Применим описанную выше схему к простому решению граничной задачи (3.1), $(3.12)$ :

$$
u^{0}(n)=2 n \ln r .
$$

Линейные задачи (3.3) и (3.8) с коэффициентами, заданными формулой (4.1), легко решаются и дают следующие выражения для $N$-солитонного случая:

$$
\begin{aligned}
& \Psi(n)=r^{-n} e^{-i \theta n} \sum_{k=1}^{N} c_{k} p_{k}^{n} r^{\left(p_{k}-1 / p_{k}\right) / 2} e^{i \theta\left(p_{k}+1 / p_{k}\right) / 2}, \\
& \widehat{\Psi}(n)=r^{n} e^{i \theta n} \sum_{k=1}^{N} d_{k} q_{k}^{n} r^{\left(q_{k}-1 / q_{k}\right) / 2} e^{-i \theta\left(q_{k}+1 / q_{k}\right) / 2},
\end{aligned}
$$


где $p_{k}, q_{k}$ - произвольные параметры, $c_{k}, d_{k}-$ постоянные коэффициенты, $k=$ $1,2, \ldots, N$.

Мы ищем решения граничной задачи (3.12), поэтому наложим дополнительные условия $(3.11),(3.13)$ на функции $\Psi(n)$ и $\widehat{\Psi}(n)$ :

$$
\left.\widehat{\Psi}(n)\right|_{r=a}=(-1)^{n} a^{2 n} e^{2 i \theta n} \Psi(n) .
$$

Используя формулы (4.2), выводим уравнение

$$
(-1)^{n} \sum_{k=1}^{N} d_{k} q_{k}^{n} a^{\left(q_{k}-1 / q_{k}\right) / 2} e^{-i \theta\left(q_{k}+1 / q_{k}\right) / 2}=\sum_{k=1}^{N} c_{k} p_{k}^{n} a^{\left(p_{k}-1 / p_{k}\right) / 2} e^{i \theta\left(p_{k}+1 / p_{k}\right) / 2}
$$

которое должно выполняться для всех $n$ и $\theta$. Таким образом, получаем следующие условия для параметров:

$$
q_{k}=-p_{k}, \quad d_{k}=c_{k} a^{p_{k}-1 / p_{k}}, \quad k=1, \ldots, N,
$$

которые соответствуют граничному условию (3.12). Далее, находим решение уравнения ГЛМ (2.7) в виде

$$
K(n, m)=-\frac{\Psi(n) \widehat{\Psi}(m)}{1+\sum_{j=n}^{\infty} \Psi(j) \widehat{\Psi}(j)},
$$

используем решения $\Psi, \widehat{\Psi}(4.2)$, условия (4.5) и из формулы (3.10) получаем решение нашей граничной задачи:

$$
u^{1}(n)=2 n \ln r-\ln (1+K(n, n))
$$

В простейшем нетривиальном случае $(N=1)$ соответствующие формулы принимают следующий вид:

$$
\begin{aligned}
\Psi(n) & =r^{-n} e^{-i \theta n} p^{n} r^{(p-1 / p) / 2} e^{i \theta(p+1 / p) / 2}, \\
\widehat{\Psi}(n) & =r^{n} e^{i \theta n} q^{n} r^{(q-1 / q) / 2} e^{-i \theta(q+1 / q) / 2}, \quad q=-p, \\
F(n, m) & =(-1)^{m} p^{n+m} e^{i \theta(p+1 / p)}, \quad K(n, n)=-\left[\left(-p^{2}\right)^{n} e^{-i \theta(p+1 / p)}+\frac{1}{1+p^{2}}\right]^{-1} .
\end{aligned}
$$

Вещественный потенциал отвечает чисто мнимым значениям параметра $p$ :

$$
u(n)=2 n \ln r-\ln (1+K(n, n)),
$$

где

$$
K(n, n)=-\left[h^{-2 n} e^{\theta(h-1 / h)}+\frac{1}{1-h^{2}}\right]^{-1}, \quad h=\operatorname{Im} p
$$




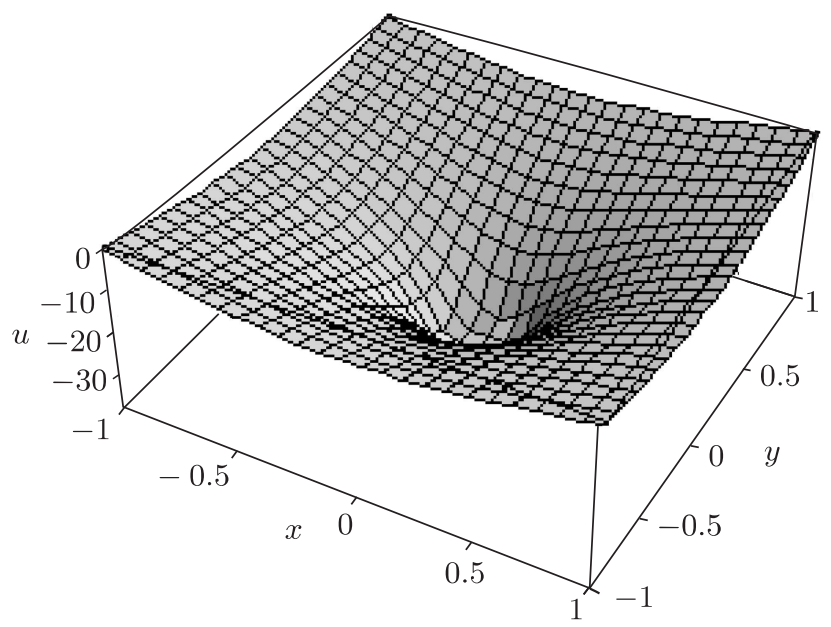

Здесь $K(n, n)$ не зависит от $r$, поэтому решение (4.8) удовлетворяет нашему граничному условию. При $0<\operatorname{Im} p<1$ оно регулярно вне окрестности точки $r=0$. Соответствующий граф для фиксированного значения индекса $n$ представлен на рисунке.

Благодарности. Автор выражает благодарность профессору И. Т. Хабибуллину за полезные советы и консультации. Работа была частично поддержана грантами Программы РАН "Фундаментальные проблемы нелинейной динамики” и РФФИ № 07-01-00081.

\section{Список литературы}

[1] А. В. Михайлов, Писъма в ЖЭТФ, 30:7 (1979), 443-448.

[2] A. V. Mikhailov, Physica D, 3:1-2 (1981), 73-117.

[3] A. Nakamura, J. Phys. Soc. Japan, 52:2 (1983), 380-387.

[4] М. В. Бабич, В. Б. Матвеев, М. А. Салль, "Биннарное преобразование Дарбу для цепочки Тоды", Вопросы квантовой теории поля и статистической физики 5, Зап. научн. сем. ЛОМИ, 145, ред. П. П. Кулиш, В. Н. Попов, ПОМИ, СПб., 1985, 34-45.

[5] И. Т. Хабибуллин, Е. В. Гудкова, Функи. анализ и его прил., 38:2 (2004), 71-83.

[6] M. Gürses, I. T. Habibullin, K. Zheltukhin, J. Math. Phys., 48:10 (2007), 102702, 16 pp.

[7] В.Э. Адлер, И. Т. Хабибуллин, А. Б. Шабат, ТМФ, 110:1 (1997), 98-113. 